\title{
A high-throughput method for combinatorial screening of metal nanoparticles using x-ray pair distribution function analysis
}

\author{
Songsheng Tao ${ }^{1}$, Samira Shiri ${ }^{2}$, Dan Kurtz ${ }^{2}$, Nate James Cira², Bryan Hunter ${ }^{2}$, Simon J. L. Billinge ${ }^{1,3}$ \\ ${ }^{I}$ Department of Applied Physics and Applied Mathematics, Columbia University, New York, NY, USA; \\ ${ }^{2}$ Rowland Institute, Harvard University, Cambridge, MA, USA; \\ ${ }^{3}$ Condensed Matter Physics and Materials Science Department, Brookhaven National Laboratory, Upton, NY, USA
}

sb2896@columbia.edu

High-throughput synthesis using inkjet printing allows the deposition of hundreds of nanoparticle compositions on a single substrate. It also introduces the challenge to characterize the phases and structures of these nanoparticles in an automated high-throughput way. Here, we develop a method to screen hundreds of nanoparticle combinations, collect the x-ray diffraction image, transfer the data to pair distribution functions and analyse the phase information automatically. It has been successfully applied to characterize the phase compositions and atomic structures in an iron, nickel, cobalt nanoparticle array. Combining this method with inkjet printing and optical screening, it is possible to achieve the fully automated high-throughput searching for the optimal combinatorial nanoparticle catalysts.
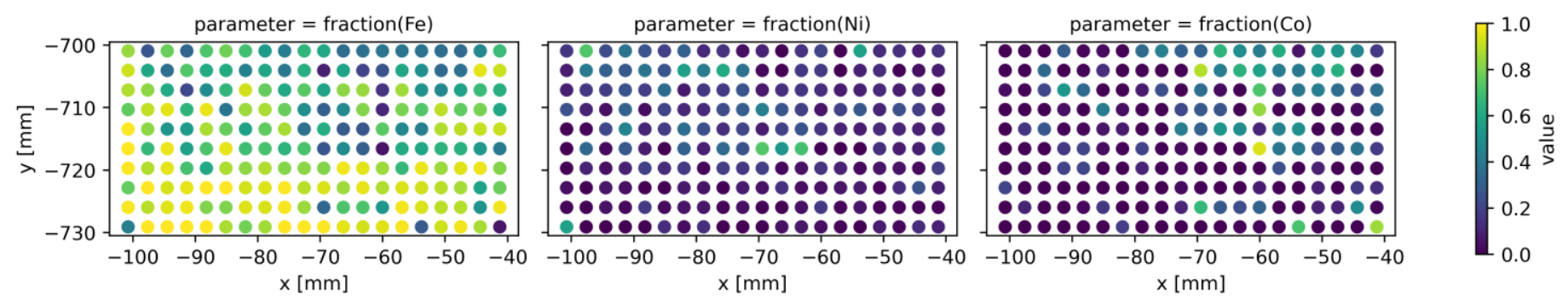

Figure 1. The molar fraction of iron, nickel, cobalt nanoparticles in an array. The $\mathrm{x}$ and $\mathrm{y}$ are the horizontal and vertical positions on the substrate, the circles represent the iron, nickel, cobalt nanoparticle combinations in an array and the colour of one circle indicates the fraction of one phase of metal nanoparticle in that combination.

Keywords: x-ray pair distribution function analysis, high-throughput, nanoparticles

Billinge's and Tao's effort on PDF analysis, modelling and writing was supported by U.S. Department of Energy, Office of Science, Office of Basic Energy Sciences (DOE-BES) under contract No. DE-SC0012704. 